Supporting Information

\title{
Self-Healing and Elastic Triboelectric Nanogenerators for Muscle Motion Monitoring and Photothermal Treatment
}

\author{
Dan Yang,$+\S$ Yufeng Ni, ${ }^{\dagger}$ Xinxin Kong, ${ }^{\dagger}$ Shuyao Li, $\|$ Xiangyu Chen, $\Perp$ Liqun \\ Zhang, ${ }^{\ddagger}$ and Zhong Lin Wang ${ }^{*} »$
}

'Beijing Key Lab of Special Elastomeric Composite Materials, Department of Material Science and Engineering, Beijing Institute of Petrochemical Technology, Beijing 102617, P. R. China

\#Department of Materials Science and Engineering, Beijing University of Chemical Technology, Beijing 100029, P. R. China

${ }^{\S}$ School of Material Science and Engineering, Georgia Institute of Technology, Atlanta, GA 30332-0245, USA

"Beijing Key Laboratory of Micro-nano Energy and Sensor, Beijing Institute of Nanoenergy and Nanosystems, Chinese Academy of Sciences, Beijing 100083, P. R. China

${ }^{*}$ Corresponding author.

E-mail: zhong.wang@mse.gatech.edu (Z. L. Wang). 
Table S1. Comparison of the electrical output performance between the original and self-healed MF-TENGs.

\begin{tabular}{ccc}
\hline & Original & Self-healed \\
\hline$I_{s c}(\mu \mathrm{A})$ & 7.98 & 6.28 \\
$Q_{s c}(\mathrm{nC})$ & 78.34 & 62.53 \\
$V_{o c}(\mathrm{~V})$ & 38.57 & 37.17 \\
\hline
\end{tabular}

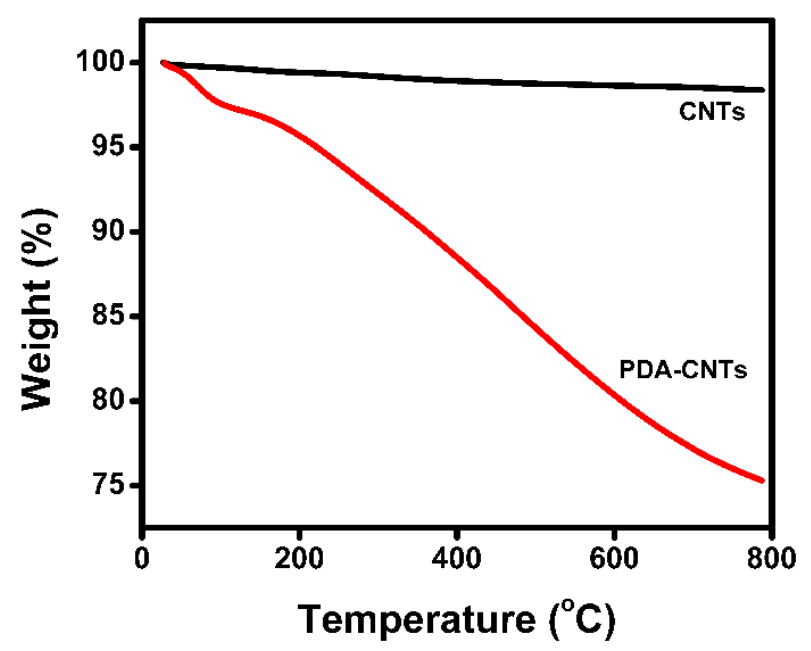

Supplementary Figure S1. TGA curves of the pristine CNTs and PDA-CNTs. 

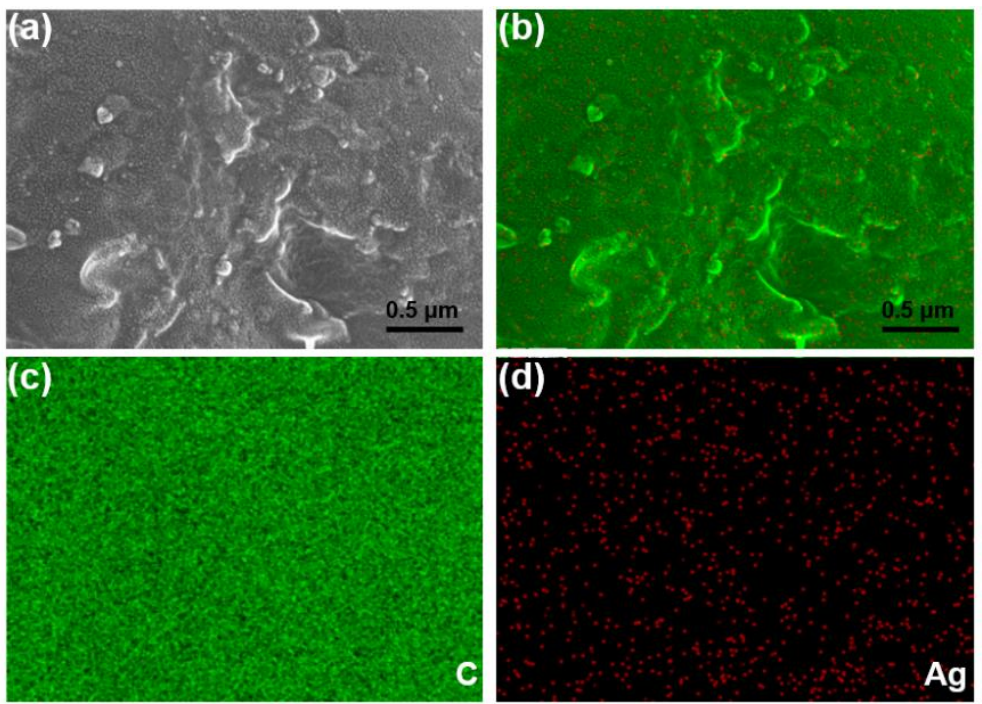

Supplementary Figure S2. (a) SEM image of the Ag-PDA-CNTs/PVA hydrogel. The EDS elemental mappings for Ag-PDA-CNTs/PVA hydrogel: (b) C and Ag, (c) C, and (d) Ag.

(a)

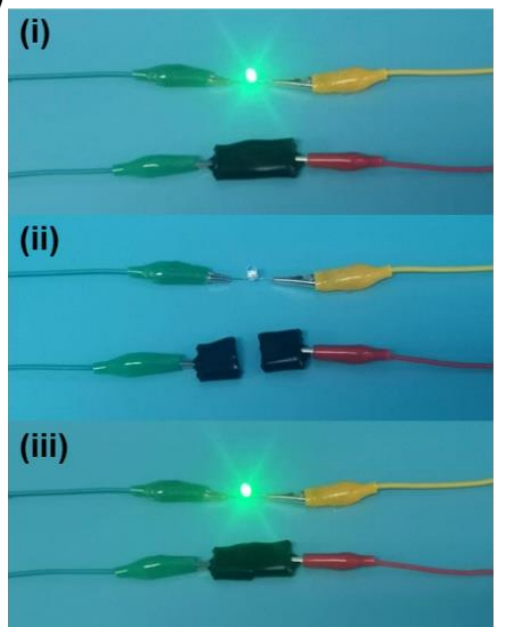

(b)

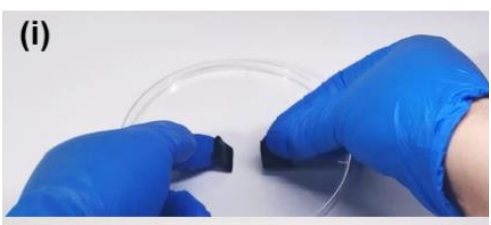

(ii)

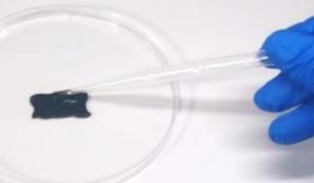

(iii)

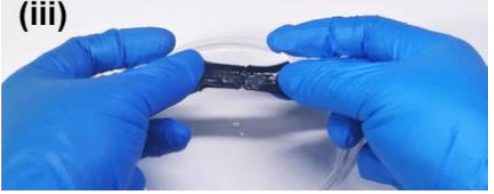

Supplementary Figure S3. (a) Optical image of (i) the original PDA-CNTs/PVA hydrogel, (ii) the cut PDA-CNTs/PVA hydrogel, and (iii) the self-healed PDA-CNTs/PVA hydrogel connecting a LED within the same circuit; (b) Digital images of (i) the cut PDA-CNTs/PVA hydrogel, (ii) the self-healed PDA-CNTs/PVA hydrogel in water environment, and (iii) the stretching self-healed PDA-CNTs/PVA hydrogel. 

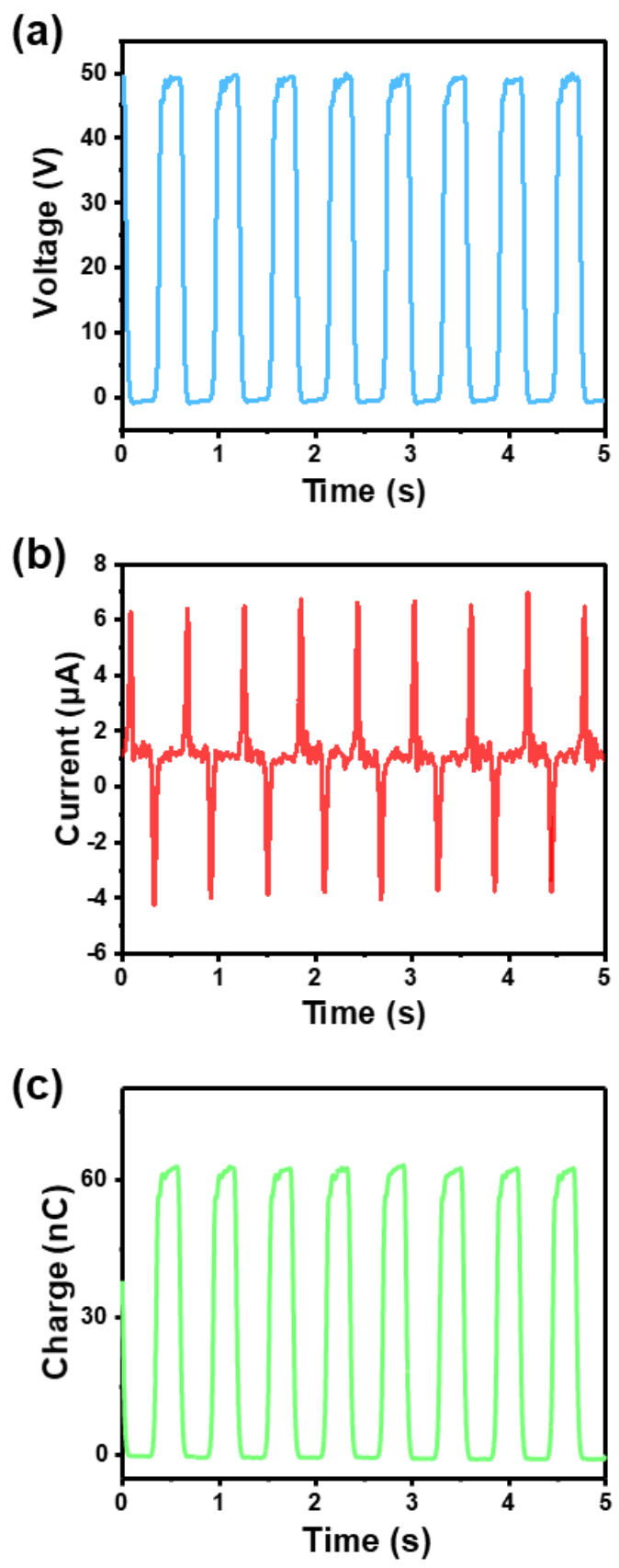

Supplementary Figure S4. (a) $V_{\mathrm{oc}}$, (b) $I_{\mathrm{sc}}$, and (c) $Q_{\mathrm{sc}}$ of the self-healing silicone elastomer. 

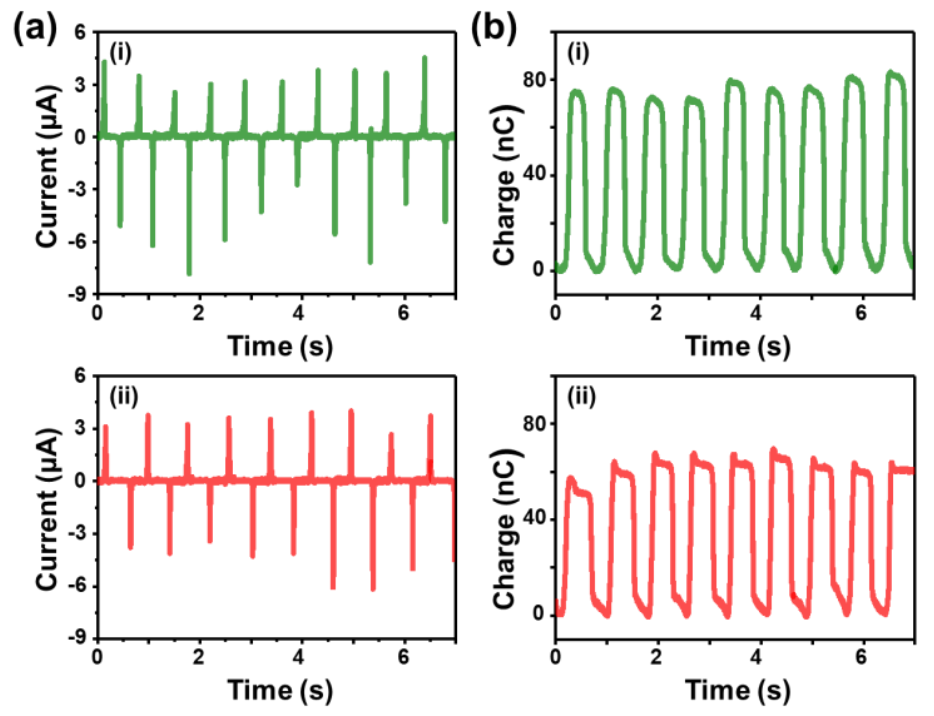

Supplementary Figure S5. (a) $I_{\mathrm{sc}}$ of (i) the original and (ii) self-healed MF-TENGs; (b) $Q_{\text {sc }}$ of (i) the original and (ii) self-healed MF-TENGs. 

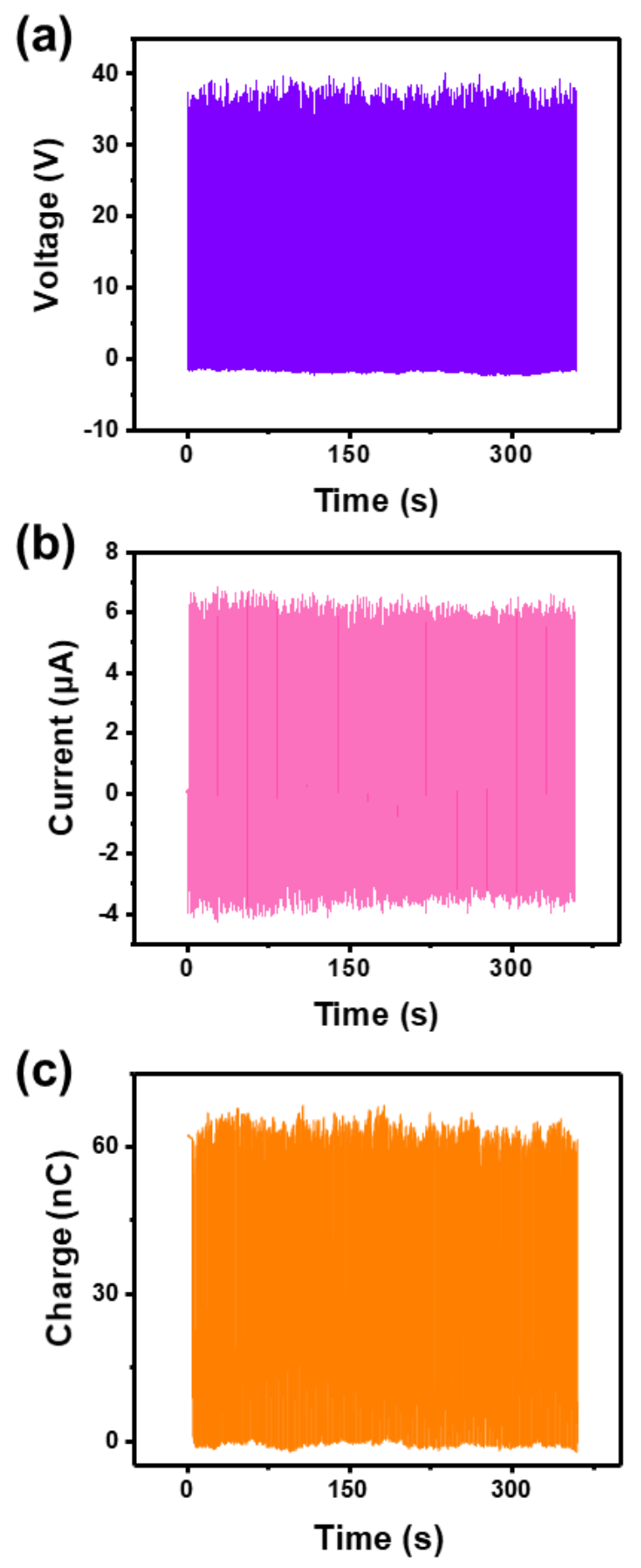

Supplementary Figure S6. (a) $V_{\mathrm{oc}}$, (b) $I_{\mathrm{sc}}$, and (c) $Q_{\mathrm{sc}}$ of the MF-TENG over 500 cycles. 


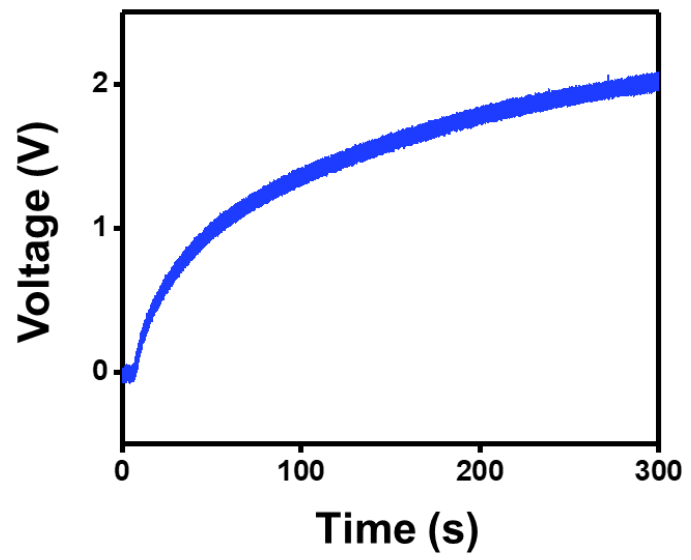

Supplementary Figure S7. The measured voltage of a $2.2 \mu \mathrm{F}$ capacitor charged by MF-TENG.

(a)

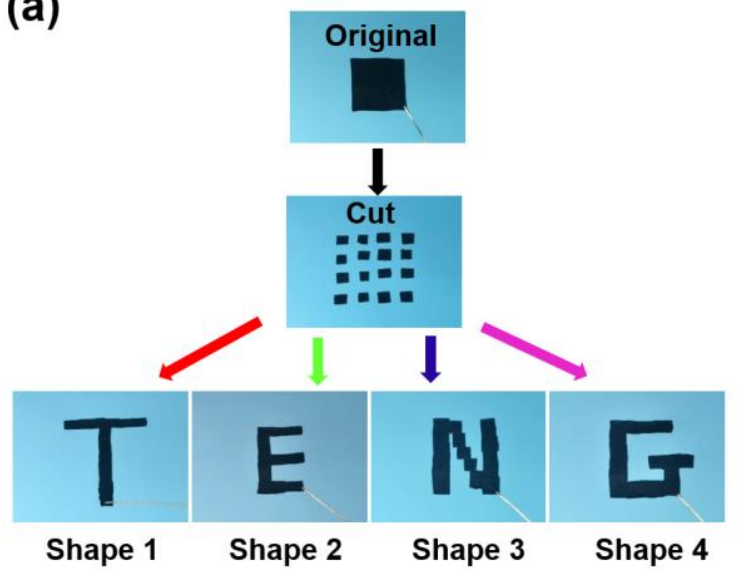

(b)

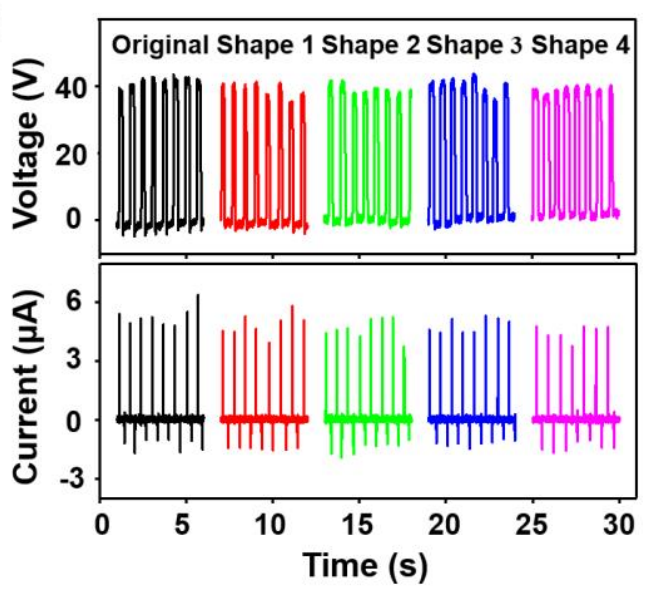

Supplementary Figure S8. (a) Optical images of the shape tunability of the MF-TENG; (b) $V_{\mathrm{oc}}$ and $I_{\mathrm{sc}}$ of the MF-TENG with different shapes. 

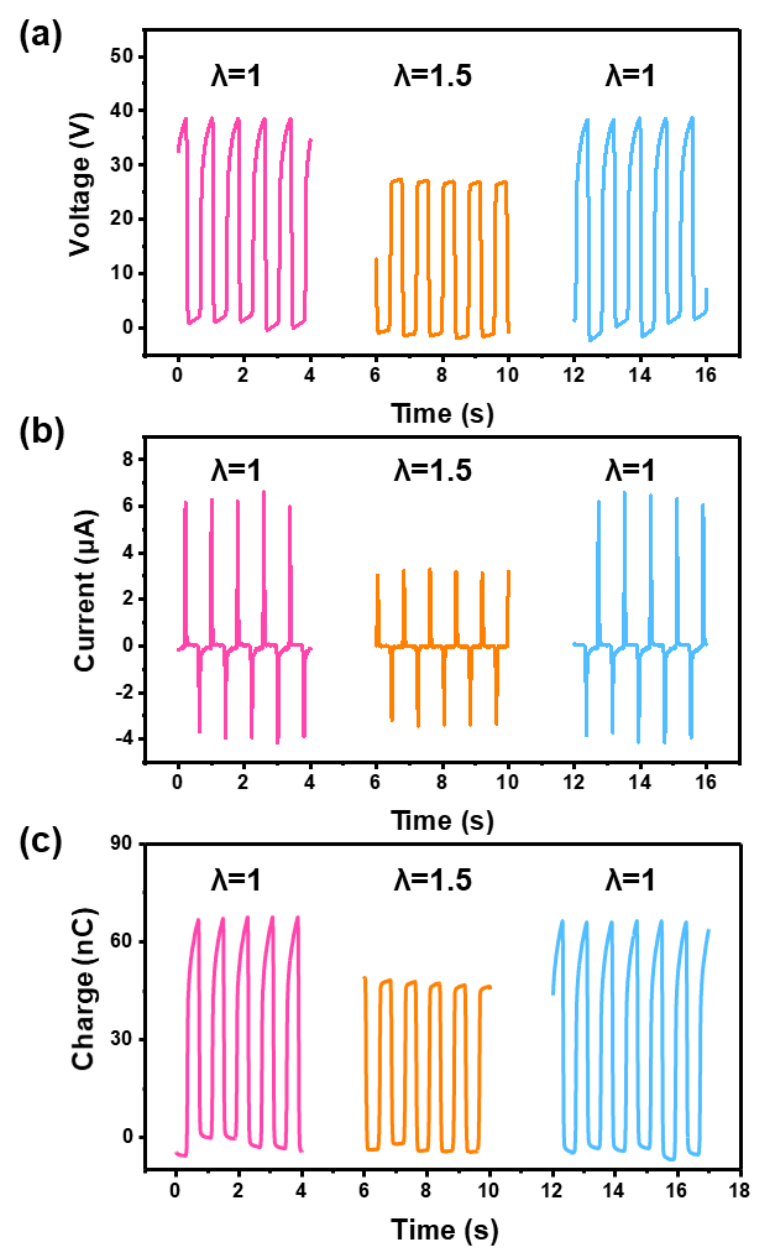

Supplementary Figure S9. (a) $V_{o c}$, (b) $I_{\mathrm{sc}}$, and (c) of the MF-TENG under different stretch ratios. 

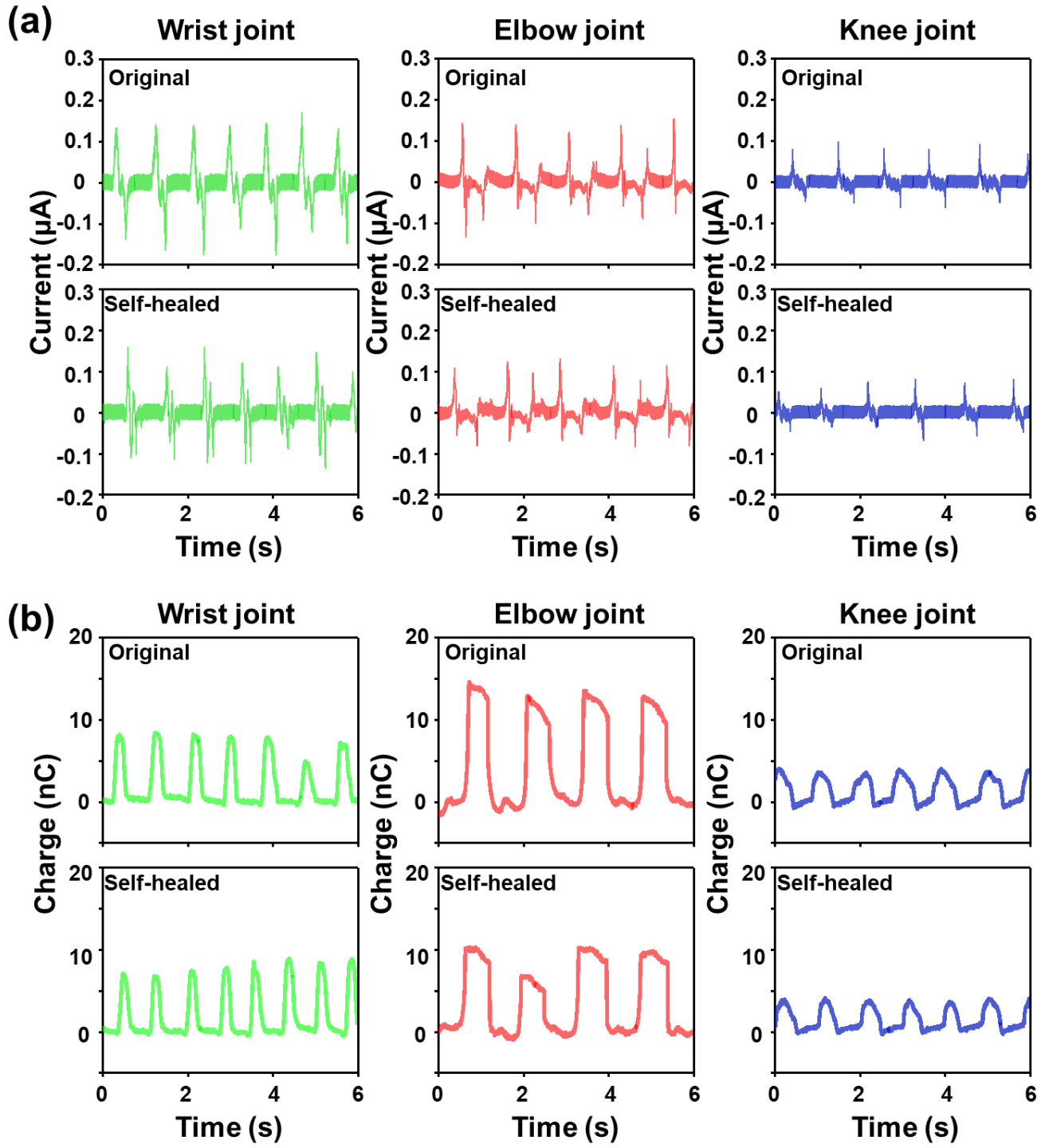

Supplementary Figure S10. (a) $I_{\mathrm{sc}}$ and (b) $Q_{\mathrm{sc}}$ of the original and self-healed MF-TENG for the wrist, elbow, and knee joint motions at the ultimate bending angle, respectively. 


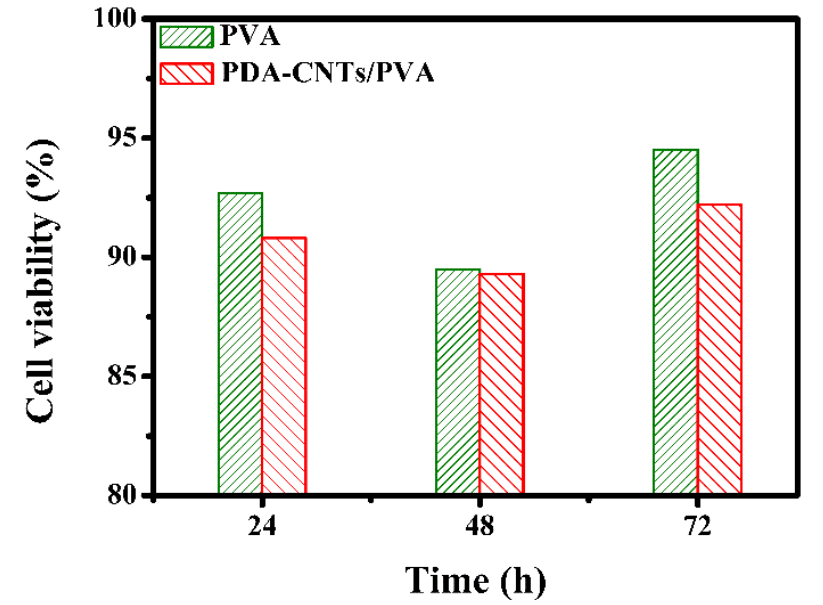

Supplementary Figure S11. Cell viability of PVA and the PDA-CNTs/PVA hydrogel. 